\title{
The differential expression of male sexual behavior in the Lewis, Fischer and Sprague-Dawley rat strains
}

\author{
Zachary E. Hurwitz • Anthony L. Riley
}

Published online: 25 November 2010

(C) The Psychonomic Society, Inc. 2010

\begin{abstract}
Several indices of sexual behavior were characterized for male rats of the Lewis (LEW), Sprague-Dawley (SD) and Fischer (F344) strains. Males were permitted access to a receptive female of their own strain for five 50min sessions every fifth day. LEW males emitted longer latencies (first mount, intromission, ejaculation and postejaculatory interval) and lower frequencies (mounts, intromissions, ejaculations, intromission ratio) relative to F344 males, with the SD strain in general, lying intermediate. Following this assessment, males of each strain were randomly assigned to females of their own or another strain to ascertain whether differences were due to differential female receptivity. Although there were no significant differences during this phase indicative of differential receptivity, the sample size may preclude a definitive interpretation of such results. The data were discussed in the context of other behavioral differences between the strains and the possible neurobiological mechanisms underlying these differences.
\end{abstract}

Keywords Sexual behavior - Strain differences - Lewis . Fischer. Sprague-Dawley

\section{Introduction}

The Lewis (LEW) and Fischer (F344) inbred rat strains are widely used animal models for examining possible genetic influences underlying the differential expression of a host

Z. E. Hurwitz $(\bowtie) \cdot$ A. L. Riley

Psychopharmacology Laboratory, Department of Psychology,

American University,

4400 Mass. Ave., NW,

Washington, DC 20016, USA

e-mail: zh7104a@student.american.edu of behavioral (Chaouloff, Kulikov, Sarrieau, Castanon, \& Mormede, 1995; Glowa, Geyer, Gold, \& Sternberg, 1992a), physiological (Guitart, Kogan, Berhow, Terwilliger, Aghajanian, \& Nestler, 1993; Marissal-Arvy, Gaumont, Langlois, Dabertrand, Bouchecareilh, Tridon et al., 2007) and neurochemical (Beitner-Johnson, Guitart, \& Nestler, 1991; Guitart, Beitner-Johnson, Marby, Kosten, \& Nestler, 1992) endpoints. Although these strains were initially examined in relation to their differences in immune, inflammatory and stress reactivity (Dhabhar, McEwen, \& Spencer, 1993; Dhabhar, Miller, McEwen, \& Spencer, 1995; Sternberg, Hill, Chrousos, Kamilaris, Listwak, Gold et al., 1989), they have also been reported to differ across a number of behavioral assays of drug use and abuse, e.g., drug self-administration (SA; Freeman, Kearns, Kohut, \& Riley, 2009; Kosten, Miserendino, Haile, DeCaprio, Jatlow, \& Nestler, 1997; Suzuki, Otani, Koike, \& Misawa, 1988), conditioned place preferences (see reviews Tzschentke, 1998, 2007), and conditioned taste aversion learning (Davis, Roma, Dominguez, \& Riley, 2007; Kosten, Miserendino, Chi, \& Nestler, 1994; Roma, Flint, Higley, \& Riley, 2006).

In addition to the abovementioned differences in a host of physiological and behavioral assays, the LEW and F344 strains have recently been reported to differ on a variety of other characteristics, including social behaviors. Berton et al. (1997), for example, compared several inbred rats (including the F344 and LEW strains) for their behavioral reactivity in various social and nonsocial settings. In a neutral environment, LEW and F344 strains had similar durations of aggressive interactions and frequencies of aggressive allogrooming, but they differed in their mean numbers of various behavioral postures (e.g., biting attacks, on top, fighting (LEW > F344) and boxing (LEW < F344). In an aversive environment, the strains did not differ in the 
number of squares crossed, but they did differ in the mean interaction times (LEW > F344). Assessments of aggression revealed that few LEW and F344 animals (16\%) initiated a fight with the intruder and there were no strain differences (in total number of fights) among those animals that did fight (Berton, Ramos, Chaouloff, \& Mormde, 1997).

More recently, Siviy, Love, DeCicco, Giordano, and Seifert (2003) extended this analysis of strain differences in social behavior by assessing playfulness (indexed by frequency of nape contacts and pins, and the probability of responding to nape contacts with a partial rotation, a complete rotation or an evasion) in juvenile LEW and F344 animals. While the LEW strain emitted more nape contacts and pins and responded more often to a nape contact with a complete rotation, F344 animals were more likely to respond to nape contacts with an evasion; there was no difference in the probability of responding to nape contact with a partial rotation (Siviy et al., 2003). Differences in social behavior between the F344 and LEW strains are not limited to paired conspecific interactions (aggression and playfulness). Specifically, Gomez-Serrano, Sternberg, and Riley (2002) measured various indices of maternal behavior (latency to first pup retrieved, latency to retrieve the entire litter, time spent crouching and the number of pups over which crouching occurred) in dams of the LEW and F344 strains and found that the LEW dams retrieved their first pup and their entire litter faster relative to the F344 strain. In addition, LEW animals spent more time crouching and crouched over significantly more pups than the F344 strain (Gomez-Serrano et al., 2002; Siviy et al., 2003).

Although LEW and F344 rats have been behaviorally characterized for a number of social behaviors, there presently are no reports of sexual behavior in these two strains. Given that such behavior are well characterized for a variety of outbred strains (Everitt, 1990; Hull \& Dominguez, 2007; Pfaus, Damsma, Nomikos, Wenkstern, Blaha, Phillips et al., 1990), procedures and behavioral baselines are established that allow for its determination and comparison in the F344 and LEW rats. In an effort to further characterize these strains, the following experiment examined sexual behavior of the F344 and LEW strains (as well as outbred Sprague-Dawley (SD) rats) to assess possible differences.

\section{Method}

\section{Subjects}

The subjects were 24 male ( 8 LEW, 8 SD, 8 F344) and 18 female (6 LEW, 6 SD, 6 F344) experimentally naïve rats. At the start of the experiment, males and females were approximately 70 days old and weighed $300-400$ and 175
200 g, respectively. Procedures recommended by the National Research Council (1996), the Committee on Guidelines for the Care and Use of Animals in Neuroscience and Behavioral Research (2003) and the Institutional Animal Care and Use Committee at American University were followed at all times. Animals were handled daily approximately two weeks prior to the initiation of the study to limit the effects of handling stress during testing.

\section{Apparatus}

All subjects were maintained in individual polycarbonate bins $(26 \times 48 \times 21 \mathrm{~cm})$ with food and water available ad libitum. The animals were maintained on a reverse 12:12 light-dark cycle (lights off at $1100 \mathrm{~h}$ ) and at an ambient temperature of $23^{\circ} \mathrm{C}$. All behavioral testing took place under $25 \mathrm{~W}$ red lights.

\section{Drugs}

For stimulus females (see below), estradiol benzoate (Sigma, St. Louis, MO) was suspended in a concentration of $25 \mu \mathrm{g} / \mathrm{rat}$ in corn oil and administered subcutaneously (SC) at a dose of $0.2 \mathrm{ml} / \mathrm{rat}$. Progesterone (Sigma, St. Louis, MO) was also suspended in corn oil at $1 \mathrm{mg} / \mathrm{rat}$ and injected SC at a dose of $0.2 \mathrm{ml} / \mathrm{rat}$ (Agmo \& Berenfeld, 1990; Camacho, Sandoval, \& Paredes, 2004; Martinez \& Paredes, 2001).

Procedure: within-strain copulatory exposure

\section{Phase I: surgery}

Stimulus female rats were bilaterally ovariectomized (OVX) when they reached approximately 175-200 g in order to prevent pregnancy and to control sexual receptivity. All ovariectomies were performed in accordance with aseptic surgical procedures. Animals were placed under deep anesthesia using a standard Ketamine $(80 \mathrm{mg} / \mathrm{kg})$ and Xylazine $(12 \mathrm{mg} / \mathrm{kg})$ mixture. Deep anesthesia was confirmed by a lack of corneal reflex and no response to tail pinch. After deep anesthesia was confirmed, incisions on both sides of the female were made to remove both of the ovaries. To do so, two dorsal to the midline incisions were made on each side of the body: one in the skin and one in the abdominal wall; the ovaries were then removed. This was done by tying non-absorbable suture around the stump of the ovaries, and then mechanically ablating the ovaries above the tied suture. The abdominal wall was then closed with sterile non-absorbable suture material (Chromic Gut; Ethicon, $27 \mathrm{~mm}$ ), and skin incisions were closed using sterile surgical staples which were removed 10 days after surgery. Once a day for 2 days following the surgery, 
females were given Ketaprofen $(5 \mathrm{mg} / \mathrm{kg})$ via intraperitoneal (IP) injection to alleviate post-operative pain or distress. The OVX female rats were then given 14 days to recover after surgery before using them as stimulus females (see below). Approximately $50 \mathrm{~h}$ post-operation, females started to receive replacement hormone therapy (see below), which was required to bring the females into behavioral estrous.

\section{Phase II: assessment of sexual behavior}

During this phase, males were given access to a receptive female of their own strain for five sessions every 5 th day. Prior to making each assessment, females were given two injections to bring them into behavioral estrous. First, it was injected SC with estradiol benzoate and then $48 \mathrm{~h}$ later injected SC with progesterone. Four hours following this second injection, each female was exposed to an untested male and assessed for both female sexual proceptivity (e.g., darting, hopping) and receptivity (e.g., displaying lordosis). This assessment was done in the dark phase of the lightdark cycle and took place in the male's homecage. Females were only used as stimuli if they expressed both proceptive and receptive behaviors, i.e., if either aspect of female sexual behavior was absent, that female was not used as a stimulus for further testing. After confirming the presence of female sexual behavior, the analyses of male sexual behavior in the abovementioned strains commenced. The procedure consisted of placing a sexually responsive female in the male's homecage for $50 \mathrm{~min}$, and upon completion of each session the female was returned to its own homecage. During this session, the interaction was digitally recorded and later scored for all relevant indices of sexual behavior by an experienced observer who was blind to the strains being tested.

Although sexual behavior has not been systematically examined in the LEW and F344 strains, such behavior in outbred rats (and in other rodent strains; see Everitt, 1990; Hull \& Dominguez, 2007) is well documented and characterized. For example, during a sexual encounter male rats engage in three stereotypically defined behaviors: mounting, intromission and ejaculation. A mount is defined as male grasping the female's flanks with his forepaws and thrusting to attempt penetration of the vagina. Intromission is defined as penetrating the female's vagina. Finally, an ejaculation is distinct from either a mount or intromission in that a male emits a longer, deeper thrust, followed by a slower dismount. An ejaculation typically occurs after approximately 7-10 intromissions. Once the male ejaculates, there is a post-ejaculatory interval (PEI), during which further sexual behavior is absent. Following this period, males will again attempt to engage in copulation if a sexually receptive female is present (Hull \& Dominguez,
2007). Several indices have been used as behavioral measures of the motivation to engage in sexual behavior and sexual performance broadly defined, e.g., mount latency (ML, latency to first mount), intromission latency (IL, latency to the first intromission), ejaculation latency (EL), mount frequency (MF), intromission frequency (IF), ejaculation frequency $(\mathrm{EF})$ and intromission ratio $\{\mathrm{IR}=[\mathrm{IF} /$ $(\mathrm{IF}+\mathrm{MF}) * 100]\}$ (Bitran \& Hull, 1987).

Procedure: cross-strain copulatory exposure

Five days following the end of the within-strain copulatory exposure, cross-strain assessments began during which males of each strain were allowed access to females of the other strains (as well as their own). The frequency of mounts, intromissions and ejaculations was coded, and the frequencies emitted by the cross-strain pairs were compared to each other and to the within-strain control. The preparation and execution of this assessment was identical to the within-strain copulatory exposure procedure (see $\S 2.4)$ sessions with the exception that males $(n=6)$ of each strain (LEW, SD, F344) were randomly assigned to receive exposure to a receptive female of its own or another strain. As above, prior to making any cross-strain comparisons all females were tested for both proceptive and receptive behavior by exposure to an untested male; only females that exhibited both behaviors were used as stimuli in subsequent testing. All subjects from the within-strain copulatory exposure assessments were used for this assessment, with animals in each strain assigned to three subgroups (two cross-strain copulation pair groups $(n=3$ per group) and one within-strain copulation pair $(n=2)$. This procedure yielded the following copulation pairs (male-female): LEW-LEW, LEW-F344, LEW-SD, SD-SD, SD-F344, SD-LEW, F344-F344, F344-LEW, F344-SD. A within-strain control group for each strain was necessary to ensure that any alterations in behavior were not a result of natural changes in the expression of sexual behavior over time or repeated exposures.

\section{Statistical analysis}

Given that all indices of sexual behavior were coded over five sessions (Sessions 1-5) and across three strains (LEW, $\mathrm{SD}, \mathrm{F} 344)$, a 5 (Session) $\times 3$ (Strain) ANOVA was used to assess any strain-dependent differences for these observed behavioral indices. In the presence of a significant Session $\times$ Strain interaction, Tukey's HSD post-hoc analysis was used to evaluate multiple comparisons. In addition, a one-way ANOVA (with a between-group factor of Strain) was used to analyze average strain frequencies and/or latencies of relevant behaviors with use of Tukey's HSD post-hoc analysis where merited. One-way ANOVAs were 
used to analyze all cross-strain behavioral frequencies in Cross-strain copulatory exposure. For the mean percent of sessions on which the LEW, SD and F344 males expressed a mount, intromission or ejaculation, a one-way ANOVA was used and Tukey's HSD post-hoc analysis was performed when merited. For all statistical analyses, a significance level of $\alpha=0.05$ was used.

\section{Results}

Behavioral frequencies

\section{Mounts}

Figure 1 illustrates the frequency of a variety of sexual behaviors for the males of each strain over the five exposures to the target females. The ANOVA used to analyze the dependent variable of frequency of mounts expressed per session revealed a significant effect of Session $[F(4,84)=$ $2.950, \mathrm{p}=0.025]$ and Strain $[\mathrm{F}(2,21)=10.229, \mathrm{p}=0.001]$, but no significant Session $\times$ Strain interaction $[\mathrm{F}(8,84)=$ $1.768, \mathrm{p}=0.095]$ (Fig. 1a). Further analysis indicated that collapsed across sessions, the LEW strain emitted significantly fewer mounts relative to both the F344 $(p<0.05)$ and SD strains $(\mathrm{p}<0.05)$ with the F344 and SD strains not differing $(p>0.05)$.

\section{Intromissions}

In relation to the frequency of intromissions expressed per session (Fig. 1b), there was a significant effect of Strain $[F$ $(2,21)=17.572, p=0.000]$, but no significant effect of Session $[F(4,84)=1.474, p=0.217]$ nor a Session $\times$ Strain interaction $[F(8,84)=0.383, p=0.927]$. Similar to the mount frequency, the LEW strain collapsed across sessions emitted significantly fewer intromissions relative to the F344 and SD strains (p's $<0.05$ ) with the F344 and SD males not differing $(p>0.05)$.

\section{Ejaculations}

Results from the analysis of frequency of ejaculations per session revealed a significant effect of Strain $[F(2,21)=$ $39.032, p=0.000]$ and a significant Session $\times$ Strain interaction $[F(8,84)=3.805, p=0.001]$, but no significant effect of Session $[F(4,84)=2.253, p=0.070]$. Tukey's HSD revealed that while there were no differences between the SD and F344 strains (Fig. 1c; p's $<0.05$ ) during Sessions 1 and 2, both strains did significantly differ from LEW males given that this strain never copulated to ejaculation. During Sessions 3 and 4, all strains were significantly different from one another (p's $<$ 0.05). Specifically, the F344 males exhibited significantly more ejaculations than SD males with the LEW strain never copulating to ejaculation. By Session 5, the F344 strain was significantly different than the other strains (p's $<0.05)$ with the LEW and SD strains exhibiting no significant differences from one another.

\section{Intromission ratio}

The analysis of the intromission rate yielded a significant effect of Strain $[F(2,21)=9.897, p=0.001]$ and a significant Session $\times$ Strain interaction $[\mathrm{F}(8,84)=3.625$, $\mathrm{p}=0.001]$, but no effect of Session $[\mathrm{F}(4,84)=1.976$, $\mathrm{p}=0.106]$. Tukey's HSD indicated that during Sessions 2 and 4 the F344 strain exhibited a higher intromission ratio relative to LEW strain (p's $<0.05$ ) while on Session 3 the intromission ratio for the F344 strain was significantly higher than both the LEW and the SD strains (p's $<0.05$ ). By Session 5, all strains were significantly different from one another $(p$ 's $<0.05)$. Specifically, the intromission ratio for the LEW strain was less than that of the F344 strain with SD males being intermediate (see Fig. 1d).

Mean percent of sessions on which sexual behaviors were expressed

\section{Mounts}

The analysis of the mean percent of sessions on which males of the LEW, SD or F344 strains displayed a mount revealed a significant effect of Strain $[\mathrm{F}(2,21)=3.775, \mathrm{p}=$ $0.04]$, but no significant effect of Session $[F(4,84)=$ $0.163, \mathrm{p}=0.957]$ nor a Session $\times$ Strain interaction $[\mathrm{F}(8$, $84)=1.14, p=0.346]$. The one-way ANOVA revealed that there were on average fewer sessions during which LEW males expressed mounting behavior relative to the F344 strain $(\mathrm{p}=0.045)$, while comparisons between the SD and F344 strains and SD and LEW strains did not significantly differ ( $p$ 's > 0.05; see Table 1).

\section{Intromissions}

The analysis of the mean percent of sessions on which males of the LEW, SD or F344 strains displayed an intromission revealed a significant effect of Strain [F (2, $21)=4.957, p=0.017]$, but no significant effect of Session $[F(4,84)=0.629, p=0.643]$ nor a Session $\times$ Strain interaction $[\mathrm{F}(8,84)=1.455, \mathrm{p}=0.186]$. On average, the percent of sessions on which LEW males expressed an intromission was significantly less than both the F344 $(\mathrm{p}=0.016)$ and SD strains $(\mathrm{p}=0.044)$ while a comparison of the mean percent of sessions for the F344 and SD strains revealed no significant difference $(p=0.892$; see Table 1). 

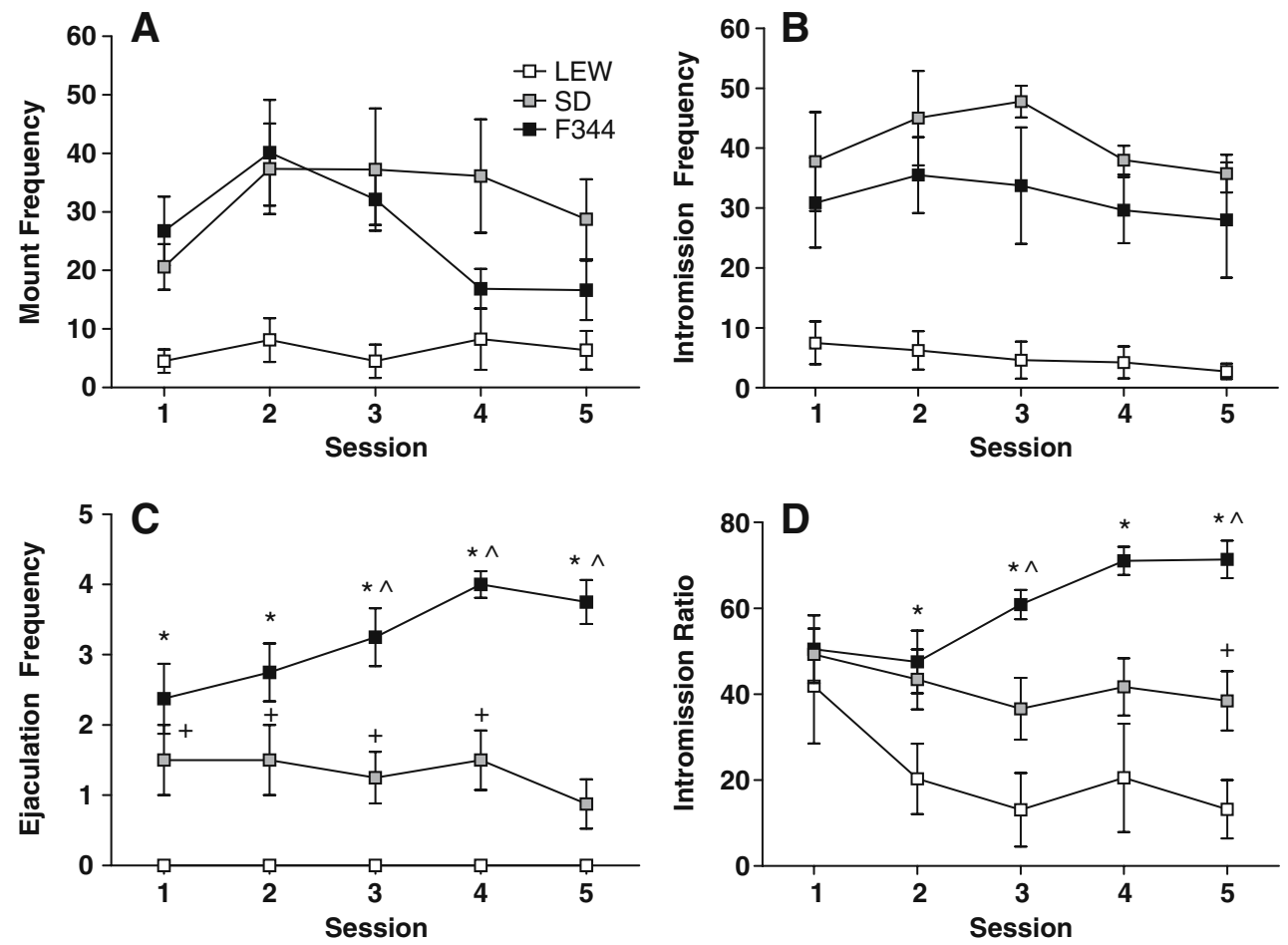

Fig. 1 The mean frequency ( \pm SEM) of mounts (a), intromissions (b), ejaculations (c) and the intromission ratio (d) for the LEW, SD and F344 males across each of the five copulatory sessions. There was a significant Session effect (such that strains emitted fewer mounts by the last session compared to the first) and Strain effect (where F344/ SD males emitted more mounts relative to LEW male $(\mathrm{p}<0.05)$ but did not differ from one another $(p>0.05))$. There was a significant

\section{Ejaculations}

Strain effect for the intromission frequency such that the F344/SD males emitted more intromissions relative to the LEW $(p<0.05)$, but were not different from one another $(p>0.05)$. For the ejaculation frequency and intromission ratio, *denotes significant differences between the F344 and LEW strains, ${ }^{+}$denotes significant differences between the LEW and SD strains and denotes significant differences between SD and F344 strains $(\mathrm{p}<0.05)$

\section{Behavioral latencies}

For the mean percent of sessions on which LEW, SD or F344 males displayed an ejaculation, there was a significant effect of Strain $[F(2,21)=23.106, p=0.000]$, but no significant effect of Session $[\mathrm{F}(4,84)=1.167, \mathrm{p}=0.331]$ nor a Session $\times$ Strain interaction $[F(8,84)=1.167, p=0.329]$. On average, the mean percent of sessions on which SD males copulated to ejaculation did not differ when compared to the F344 strain $(p=0.08)$, whereas the mean percent of sessions for the LEW strain was different from both the F344 and SD strains mean percentage in that for all sessions the LEW males never copulated to ejaculation (see Table 1).

Table 1 The mean percent of sessions on which males of the LEW, SD or F344 strains expressed a mount, intromission and ejaculation. For this table, adenotes significant differences between the F344 and LEW strains and ${ }^{+}$denotes significant differences between SD and LEW $(\mathrm{p}<0.05)$

\begin{tabular}{llll}
\hline & Mounts & Intromissions & Ejaculations \\
\hline LEW & $52.5 \%{ }^{\mathrm{a}}$ & $45 \%^{\mathrm{a}_{+}}$ & $0 \%{ }^{\mathrm{a}_{+}}$ \\
F344 & $95 \%$ & $95 \%$ & $95 \%$ \\
SD & $87.50 \%$ & $87.50 \%$ & $62.50 \%$ \\
\hline
\end{tabular}

\section{First mount}

Figure 2 illustrates the latency for a variety of sexual behaviors for the males of each strain over the five exposures to the target females. Any subject that failed to express a behavior (mount, intromission, ejaculation, PEI) during testing was assigned a latency value of $3000 \mathrm{sec}$ (length of the testing session). The analysis of the latency to the first mount revealed a significant effect of Strain $[\mathrm{F}(2,21)=$ $5.786, \mathrm{p}=0.010]$, but no significant effect of Session [F (4, $84)=1.396, p=0.242]$ nor a significant Session $\times$ Strain interaction $[\mathrm{F}(8,84)=1.410, \mathrm{p}=0.204$; Fig. 2a]. Analysis of the average latency to the first mount for each strain revealed that collapsed across sessions LEW males took significantly longer than both the F344 and SD strains (p's < 0.05 ) to emit their first mount while the F344 and SD males did not differ $(\mathrm{p}>0.05)$.

\section{First intromission}

Analysis of the latency to the first intromission indicated that there was a significant effect of Session $[F(4,84)=$ 

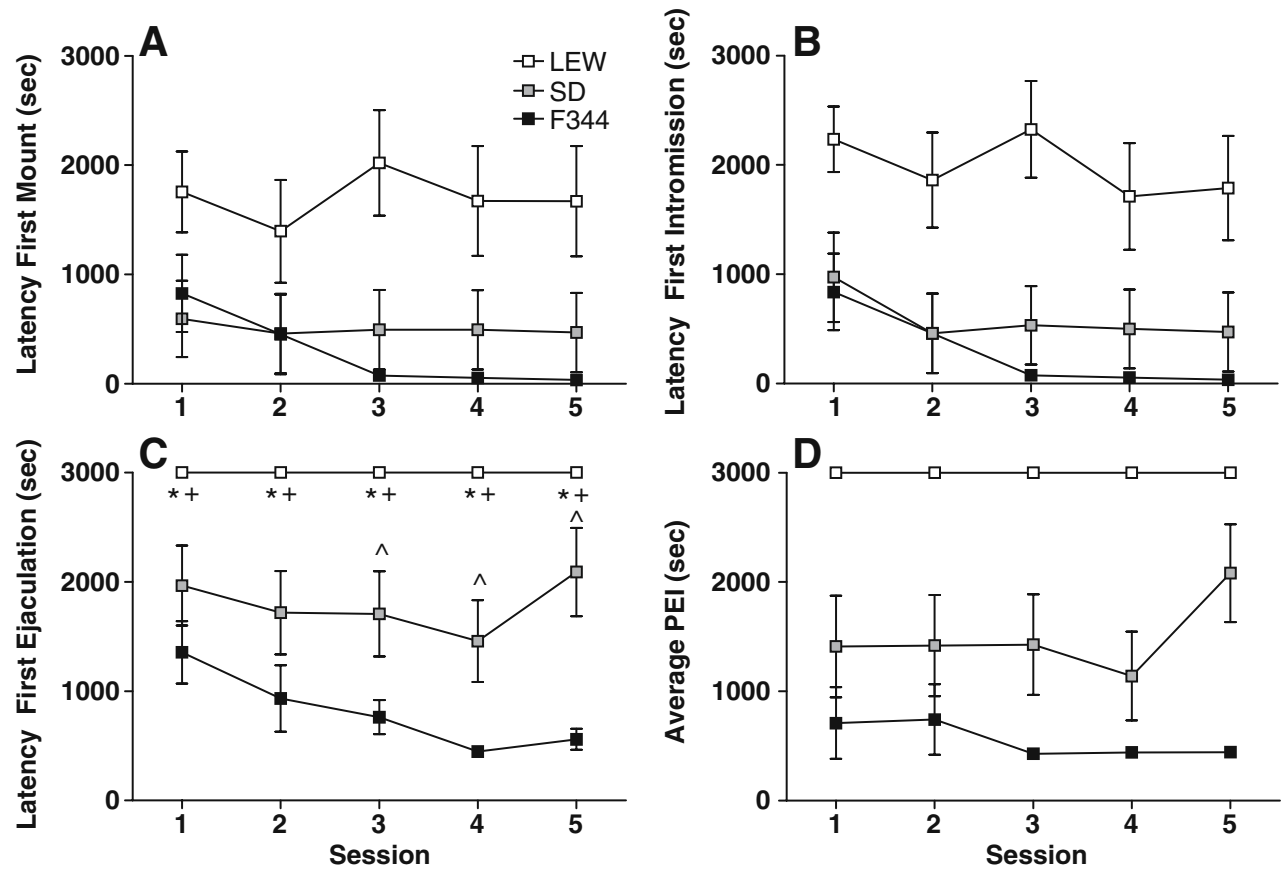

Fig. 2 The mean latency $( \pm \mathrm{SEM})$ to the first mount (a), first intromission (b), first ejaculation (c) and the post-ejaculatory interval (d) for the LEW, SD and F344 males across each of the five copulatory sessions. There was a significant effect of Strain for the latency to the first mount such that the F344/SD males had a shorter latency relative to the LEW males ( $<$ 0.05), while the F344 and SD males did not differ from one another $(p>0.05)$. Latency to the first intromission yielded significant Session and Strain effects such that

$3.914, \mathrm{p}=0.006]$ and $\operatorname{Strain}[\mathrm{F}(2,21)=9.069, \mathrm{p}=0.001]$, but no significant Session $\times$ Strain interaction $[\mathrm{F}(2,21)=0.826$, $\mathrm{p}=0.582$; Fig. 2b]. Similar to the previous index, analysis of the average latency to the first intromission indicated that it took significantly longer for the LEW strain to emit their first intromission relative to the F344 and SD strains (p's < 0.05), while the F344 and SD males displayed no significant differences $(p>0.05)$.

\section{First ejaculation}

For the analysis of latency to first ejaculation, there was a significant effect of Session $[\mathrm{F}(4,84)=3.464, \mathrm{p}=0.011]$ and Strain $[F(2,21)=27.848, p=0.000]$ and a significant Session $\times$ Strain interaction $[\mathrm{F}(8,84)=02.803, \mathrm{p}=0.046]$. Tukey's HSD indicated that during Sessions 3-5, the F344 strain emitted a significantly shorter latency to the first ejaculation relative to SD males with both strains differing significantly during all sessions from LEW animals given their absence of an ejaculation during testing (all p's $<0.05$; Fig. 2c).

\section{Average post-ejaculatory interval}

There was a significant effect of $\operatorname{Strain}[F(2,21)=25.810$, $\mathrm{p}=0.000]$, but no significant effect of Session $[\mathrm{F}(4,84)=$

the F344/SD males did not differ from one another $(p>0.05)$ but did significantly differ relative to the LEW animals and the strains exhibited longer latencies at the outset of testing. For the latency to the first ejaculation, *denotes significant differences between the F344 and LEW strains, ${ }^{+}$denotes significant differences between SD and LEW strains and ${ }^{\wedge}$ denotes significant differences between the F344 and SD strains $(\mathrm{p}<0.05)$

$1.492, \mathrm{p}=0.212]$ nor a significant Session $\times$ Strain interaction $[\mathrm{F}(8,84)=1.879, \mathrm{p}=0.074$; Fig. $2 \mathrm{~d}]$. When the average PEI was collapsed across sessions, LEW animals, because they failed to ejaculate at all, had a significantly longer PEI relative to the F344 strain with SD males falling intermediate (p's $<0.05$ ).

\section{Cross-strain copulatory exposure}

For each strain, the behavioral frequencies of mounts, intromissions and ejaculations were compared between males paired with females of their own (within) and other (cross) strains. There were no significant differences in the frequencies of mounts $[\mathrm{F}(2,7)=0.989, \mathrm{p}=0.435$; Fig. 3a] or intromissions $[\mathrm{F}(2,7)=1.376, \mathrm{p}=0.334$; Fig. $3 \mathrm{~b}]$ when comparing LEW males paired with LEW females to that of LEW males paired with either a SD or F344 female. Analysis of their ejaculation frequency was not possible given that no LEW male (regardless of the strain of the female copulation partner) copulated to ejaculation in the allotted time (Fig. 3c). SD males did not significantly differ in regards to their mount $[\mathrm{F}(2,7)=1.502, \mathrm{p}=0.308$; Fig. 3a], intromission $[\mathrm{F}(2,7)=1.420, \mathrm{p}=0.325$; Fig. 3b] or ejaculation $[F(2,7)=1.625, p=0.286$; Fig. $3 c]$ frequencies when comparing SD males paired with SD females to that 
Fig. 3 The mean frequency ( \pm SEM) of mounts (a), intromissions (b) and ejaculations (c) for the LEW, SD or F344 males that were paired with females of their own or another strain. The color of the bar denotes the strain of male used (white $=$ LEW male, grey $=\mathrm{SD}$ male, black $=$ F344 male), while the labels on the $\mathrm{X}$-axis denote the strain of the female paired with that male. There were no significant differences in the behavioral frequencies when males of the LEW, SD or F344 strains were paired with a female of their own or another strain $(\mathrm{p}>0.05)$

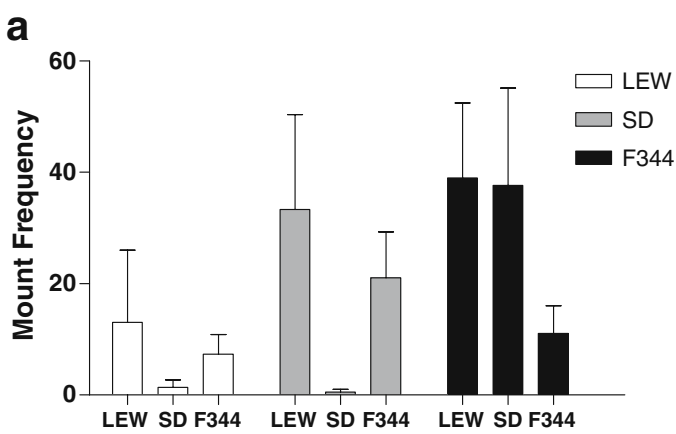

b

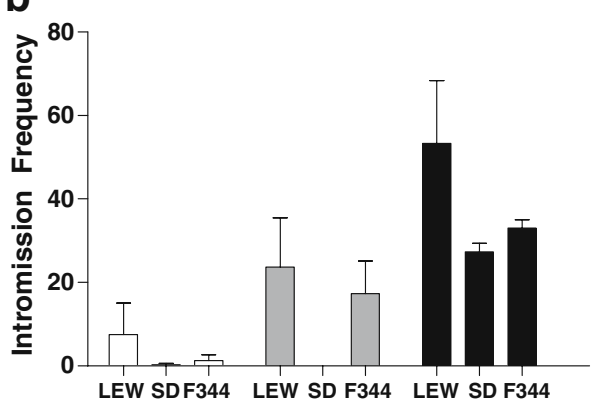

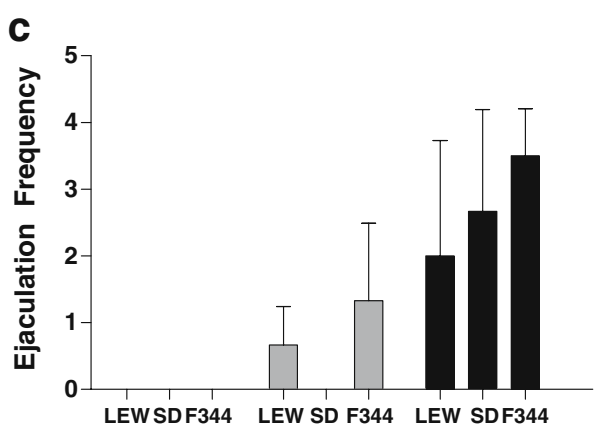

of SD males paired with either a LEW or F344 female. Finally, there were no significant differences in mount $[\mathrm{F}(2$, $7)=0.945, p=0.449$; Fig. 3a], intromission $[F(2,7)=$ $1.964, p=0.235$; Fig. 3b] or ejaculation $[F(2,7)=0.606$, $\mathrm{p}=0.581$; Fig. $3 \mathrm{c}]$ frequencies when comparing F344 males paired with F344 females to that of F344 males paired with either a SD or LEW female.

\section{Discussion}

In the present experiment, males of the LEW, SD and F344 rat strains were paired with females of their own strain and the behavioral frequencies and latencies of a variety of sexual behaviors were assessed. As described, analyses of both the average mount and intromission frequencies revealed that the LEW strain differed significantly from the F344 and SD strains (LEW < F344/SD ), while the average latency to the first mount and latency to the first intromission yielded an opposite effect (LEW > F344/SD). Further, the intromission ratio was greater for F344 males relative to LEW males at Sessions 2 and 4 and greater than both LEW and SD males on Session 3. By Session 5, all strains differed from one another. In general, F344 rats were significantly different from the SD rats for the indices of ejaculation frequency (F344 > SD), latency to first ejaculation (F344 < SD) and the average PEI length (F344 $<\mathrm{SD}$ ), while, in general, both strains were significantly different than LEW animals. Finally, the mean percentage of sessions on which LEW males engaged in sexual behavior was lower relative to males of the F344 and SD strains. Although little has been reported about the sexual behavior of the F344 and LEW strains, it is interesting that Harlan Laboratories ${ }^{\mathrm{TM}}$ (which commercially breeds the LEW and F344 strains) report that the LEW strain has a high rate of sterility, and LEW females have longer time intervals between litters.

The fact that differences exist in sexual behavior adds to the expanding list of behavioral differences between the LEW and F344 rats. As noted above, differences between the two strains have also been reported for a host of behaviors, including (but not limited to) juvenile play (Siviy et al., 2003), maternal behavior (Gomez-Serrano et al., 2002), social responsiveness (Berton et al., 1997) and drug reactivity (Riley, Davis, \& Roma, 2009) (for a review see Kosten \& Ambrosio, 2002). The present data also add to the complexity of the reported differences in that the direction of these differences varies greatly. For example, in the present experiment, the F344 strain displayed behaviors suggestive of a more robust sexual response, e.g., reduced latencies and greater frequencies of behavior (as well as the presence of ejaculations). Consistent with these findings, F344 rats also develop schedule-induced polydipsia at a faster rate and display greater overall levels of this behavior at asymptote (see DeCarolis, Myracle, Erbach, Glowa, Flores, Riley et al., 2003). Interestingly, in other behavioral assays (both social and non-social), it is the LEW strain that expresses more robust behaviors, e.g., expression of juvenile play (Siviy et al., 2003) and maternal behavior (Gomez-Serrano et al., 2002). For instance, Siviy et al. (2003) reported that juvenile F344 rats emitted fewer nape contacts and pins, were less likely to initiate playful activity 
and were more likely to evade an attempt at a playful interaction initiated by a partner relative to the LEW strain. In yet other assays (e.g., aggression, see Berton et al., 1997; stress reactivity, Chaouloff et al., 1995; food intake, Haile \& Kosten, 2001), there are no differences between the two strains (for additional behavioral comparisons, see Kosten $\&$ Ambrosio, 2002). From comparisons across these very different designs, there is no common element suggestive of one strain being more or less reactive then the other. Such differences in these behavioral displays (and in the direction of these differences) may be a challenge in determining a common mechanism (biochemical; motivational; neuroanatomical) for differences between the two strains.

The focus of this discussion has been on the differences between the LEW and F344 strains, but it is important to note that on some of the behavioral measures differences were also evident between these two strains and the outbred control (i.e., the SD rats). The present report describes these strains as exhibiting behavior lying on a continuum with the F344 rats expressing higher frequencies and shorter latencies of sexual behavior relative to the LEW strain with SD rats being intermediate. Such a characterization has previously been described when comparing the LEW and F344 strains with outbred controls. Specifically, for several behavioral measures of stress (open-field, swim stress, restraint stress) the F344 strain is generally more responsive than LEW animals, with the SD strain being intermediate (Sternberg, Glowa, Smith, Calogero, Listwak, Aksentijevich et al., 1992). Findings by Glowa et al. (1992a; Glowa, Sternberg, \& Gold, 1992b) further demonstrated this continuum (albeit in a different direction) when acoustic-startle and tactile-startle responses were assayed with the SD males once again falling intermediate of the two inbred strains (Glowa \& Hansen, 1994; Glowa et al., 1992b). It is important to note, however, that the SD strain does not always fall intermediate of the inbred strains (see Shoaib, Schindler, \& Goldberg, 1997; Tang, Liu, Yang, \& Sanford, 2005; Varty \& Geyer, 1998), indicating that the differences reported and the direction of these differences once again are dependent upon the specific behavioral assay under consideration.

Although the differences between the LEW and F344 rat strains in sexual behavior (among others) are clear, the basis for such differences remains unknown. Several possibilities exist, however. One possibility for these reported differences concerns the specific assay of sexual behavior used in the present experiment, i.e., it may have been one that exacerbated behavioral differences not generally reported in such assessments. However, the fact that the outbred SD rats displayed behaviors comparable to those reported in other assessments with this strain suggests that the specific preparation used here was able to index sexual behavior appropriately (e.g., see Straiko, Gudelsky,
\& Coolen, 2007). Specifically, when controlling for session length, SD rats in the present study were comparable in both frequency (mount, intromission and ejaculation) and latencies (mount and ejaculation) relative to those reported by Straiko and colleagues. Although the intromission ratio calculated for Straiko's subjects was $15 \%$ lower than that presented in the current report ( $35 \%$ vs. $50 \%$, respectively), this may result from differences in the duration of exposure to receptive females. Straiko et al. (2007) were interested in the subjects copulating to a single ejaculation to use for place conditioning, and thus there was no recording of a PEI, precluding a comparison. Aside from specific reports using the SD strain, male sexual behavior has been well characterized in other outbred rodent strains (e.g., for Wistar rats see Agmo \& Berenfeld, 1990; Agmo \& Gomez, 1993; Camacho et al., 2004; Martinez \& Paredes, 2001; Paredes \& Alonso, 1997; for Long-Evans rats see Damsma, Pfaus, Wenkstern, Phillips, \& Fibiger, 1992; Pfaus et al., 1990; Robertson, Pfaus, Atkinson, Matsumura, Phillips, \& Fibiger, 1991; Westerman, Roma, Creed, Hurwitz, \& Dominguez, 2009). In general, SD males express higher frequencies and lower latencies when compared to experiments with the Wistar strain, whereas SD males emit lower frequencies and longer latencies relative to preparations using Long-Evans males. When making these comparisons, it is important to note that some procedures required animals to be sexually experienced prior to starting any behavioral recordings. For instance, in one report using Long-Evans males, each subject was given up to six sessions of free copulatory experience with a receptive female before recording behavioral indices (Robertson et al., 1991). Despite varying experimental conditions, the parameters used in the current experiment elicited sexual behavior within the usual range reported in previous assessments of sexual behavior in outbred strains.

Another possibility is that the reported differences possibly reflect differential receptivity of the females of the various strains. There are several lines of evidence against this possibility. As previously stated, females had to display both proceptive and receptive behavior when exposed to an untested male to be used as stimuli in subsequent testing. Further, at the time of testing proceptivity was monitored and all females displayed appropriate sexual behaviors. Finally, there were no significant changes in sexual behavior frequencies (mounts, intromissions and ejaculations) when cross-strain comparisons were made, although this conclusion should be interpreted with caution given the sample sizes used in the current assessment. Despite these arguments, it is nonetheless possible that female sexual behavior may have been involved in subsequent male reactivity. Specifically, sexual behavior involves a complex dynamic between the males and females. For example, receptive behaviors such as lordosis 
are a function of interactions with the male, and if the male initiates no sexual interactions in response to the female it would be unlikely that lordosis would be displayed. This, in turn, would limit the likelihood of subsequent mounts, intromissions and ejaculations. The differences between males of the different strains could be a function of an interaction of their behavior and the subsequent reactivity of the females. Accordingly, statements regarding differential male behavior must be considered in light of this dynamic with the female. Additional assessments are needed to better understand the aforementioned strain differences and determine the contribution of the female to these differences.

Another possibility for the reported strain differences in sexual behavior centers around the mesolimbic system and its role in differences in drug intake between the LEW and F344 rats (Beitner-Johnson et al., 1991; Guitart et al., 1992; Haile \& Kosten, 2001; for a review, see Kosten \& Ambrosio, 2002). The different neurobiology and activation of this area has been implicated in the high levels of acute drug intake in the LEW and F344 rats. Interestingly, this area of the brain has also been examined in relation to other motivated responses including sexual behaviors in outbred rats (see Hull \& Dominguez, 2007). For example, Pfaus et al. (1990) demonstrated that DA levels in the mesolimbic area (specifically the NAc) increase in response to copulatory exposure (see also Damsma et al., 1992). Such assessments have not been made in the LEW and F344 strains, limiting conclusions regarding the role of this area in sexual behavior of these two strains (or any reported differences). These investigations would be complemented by assessments of other neurobiological substrates reported to play roles in sexual behavior in outbred strains (for review, see Hull \& Dominguez, 2007; Kosten \& Ambrosio, 2002). These investigations would be important in determining if such differences were mediated by differences in the mesolimbic system (or other neurobiological substrates) and if these differences paralleled those reported for other differences between the two strains.

Acknowledgements This work was supported in part by a grant from the Mellon Foundation to Anthony L. Riley. Requests for reprints should be sent to Zachary E. Hurwitz, Psychopharmacology Laboratory, Department of Psychology, American University, Washington, DC 20016 (or zh7104a@student.american.edu).

\section{References}

Agmo, A., \& Berenfeld, R. (1990). Reinforcing properties of ejaculation in the male rat: Role of opioids and dopamine. Behavioral Neuroscience, 104(1), 177-182.

Agmo, A., \& Gomez, M. (1993). Sexual reinforcement is blocked by infusion of naloxone into the medial preoptic area. Behavioral Neuroscience, 107(5), 812-818.
Beitner-Johnson, D., Guitart, X., \& Nestler, E. J. (1991). Dopaminergic brain reward regions of Lewis and Fischer rats display different levels of tyrosine hydroxylase and other morphine- and cocaine-regulated phosphoproteins. Brain Research, 561(1), $147-150$.

Berton, O., Ramos, A., Chaouloff, F., \& Mormde, P. (1997). Behavioral reactivity to social and nonsocial stimulations: A multivariate analysis of six inbred rat strains. Behavior Genetics, 27(2), 155-166.

Bitran, D., \& Hull, E. M. (1987). Pharmacological analysis of male rat sexual behavior. Neuroscience and Biobehavioral Reviews, 11(4), 365-389.

Camacho, F., Sandoval, C., \& Paredes, R. G. (2004). Sexual experience and conditioned place preference in male rats. Pharmacology, Biochemistry and Behavior, 78(3), 419-425.

Chaouloff, F., Kulikov, A., Sarrieau, A., Castanon, N., \& Mormede, P. (1995). Male Fischer 344 and Lewis rats display differences in locomotor reactivity, but not in anxiety-related behaviours: Relationship with the hippocampal serotonergic system. Brain Research, 693(1-2), 169-178.

Damsma, G., Pfaus, J. G., Wenkstern, D., Phillips, A. G., \& Fibiger, H. C. (1992). Sexual behavior increases dopamine transmission in the nucleus accumbens and striatum of male rats: Comparison with novelty and locomotion. Behavioral Neuroscience, 106(1), 181-191.

Davis, C. M., Roma, P. G., Dominguez, J. M., \& Riley, A. L. (2007). Morphine-induced place conditioning in Fischer and Lewis rats: Acquisition and dose-response in a fully biased procedure. Pharmacology, Biochemistry and Behavior, 86(3), 516-523.

DeCarolis, N. A., Myracle, A., Erbach, J., Glowa, J., Flores, P., \& Riley, A. L. (2003). Strain-dependent differences in scheduleinduced polydipsia: An assessment in Lewis and Fischer rats. Pharmacology, Biochemistry and Behavior, 74(3), 755-763.

Dhabhar, F. S., McEwen, B. S., \& Spencer, R. L. (1993). Stress response, adrenal steroid receptor levels and corticosteroid-binding globulin levels - a comparison between Sprague-Dawley, Fischer 344 and Lewis rats. Brain Research, 616(1-2), 89-98.

Dhabhar, F. S., Miller, A. H., McEwen, B. S., \& Spencer, R. L. (1995). Differential activation of adrenal steroid receptors in neural and immune tissues of Sprague Dawley, Fischer 344, and Lewis rats. Journal of Neuroimmunology, 56(1), 77-90.

Everitt, B. J. (1990). Sexual motivation: A neural and behavioural analysis of the mechanisms underlying appetitive and copulatory responses of male rats. Neuroscience and Biobehavioral Reviews, 14(2), 217-232.

Freeman, K. B., Kearns, D. N., Kohut, S. J., \& Riley, A. L. (2009). Strain differences in patterns of drug-intake during prolonged access to cocaine self-administration. Behavioral Neuroscience, 123(1), 156-164.

Glowa, J. R., Geyer, M. A., Gold, P. W., \& Sternberg, E. M. (1992). Differential startle amplitude and corticosterone response in rats. Neuroendocrinology, 56(5), 719-723.

Glowa, J. R., \& Hansen, C. T. (1994). Differences in response to an acoustic startle stimulus among forty-six rat strains. Behavior Genetics, 24(1), 79-84.

Glowa, J. R., Sternberg, E. M., \& Gold, P. W. (1992). Differential behavioral response in LEW/N and F344/N rats: Effects of corticotropin releasing hormone. Progress in Neuropsychopharmacology \& Biological Psychiatry, 16(4), 549-560.

Gomez-Serrano, M. A., Sternberg, E. M., \& Riley, A. L. (2002). Maternal behavior in $\mathrm{F} 344 / \mathrm{N}$ and LEW/N rats. Effects on carrageenan-induced inflammatory reactivity and body weight. Physiology \& Behavior, 75(4), 493-505.

Guitart, X., Beitner-Johnson, D., Marby, D. W., Kosten, T. A., \& Nestler, E. J. (1992). Fischer and Lewis rat strains differ in basal levels of neurofilament proteins and their regulation by chronic 
morphine in the mesolimbic dopamine system. Synapse, 12(3), $242-253$.

Guitart, X., Kogan, J. H., Berhow, M., Terwilliger, R. Z., Aghajanian, G. K., \& Nestler, E. J. (1993). Lewis and Fischer rat strains display differences in biochemical, electrophysiological and behavioral parameters: Studies in the nucleus accumbens and locus coeruleus of drug naive and morphine-treated animals. Brain Research, 611(1), 7-17.

Haile, C. N., \& Kosten, T. A. (2001). Differential effects of D1- and D2-like compounds on cocaine self-administration in Lewis and Fischer 344 inbred rats. The Journal of Pharmacology and Experimental Therapeutics, 299(2), 509-518.

Hull, E. M., \& Dominguez, J. M. (2007). Sexual behavior in male rodents. Hormones and Behavior, 52(1), 45-55.

Kosten, T. A., \& Ambrosio, E. (2002). HPA axis function and drug addictive behaviors: Insights from studies with Lewis and Fischer 344 inbred rats. Psychoneuroendocrinology, 27(1-2), $35-69$.

Kosten, T. A., Miserendino, M. J., Chi, S., \& Nestler, E. J. (1994). Fischer and Lewis rat strains show differential cocaine effects in conditioned place preference and behavioral sensitization but not in locomotor activity or conditioned taste aversion. The Journal of Pharmacology and Experimental Therapeutics, 269(1), 137144.

Kosten, T. A., Miserendino, M. J., Haile, C. N., DeCaprio, J. L., Jatlow, P. I., \& Nestler, E. J. (1997). Acquisition and maintenance of intravenous cocaine self-administration in Lewis and Fischer inbred rat strains. Brain Research, 778(2), 418-429.

Marissal-Arvy, N., Gaumont, A., Langlois, A., Dabertrand, F., Bouchecareilh, M., Tridon, C., et al. (2007). Strain differences in hypothalamic pituitary adrenocortical axis function and adipogenic effects of corticosterone in rats. The Journal of Endocrinology, 195(3), 473-484.

Martinez, I., \& Paredes, R. G. (2001). Only self-paced mating is rewarding in rats of both sexes. Hormones and Behavior, 40(4), $510-517$.

Paredes, R. G., \& Alonso, A. (1997). Sexual behavior regulated (paced) by the female induces conditioned place preference. Behavioral Neuroscience, 111(1), 123-128.

Pfaus, J. G., Damsma, G., Nomikos, G. G., Wenkstern, D. G., Blaha, C. D., Phillips, A. G., et al. (1990). Sexual behavior enhances central dopamine transmission in the male rat. Brain Research, 530(2), 345-348.

Riley, A. L., Davis, C. M., \& Roma, P. G. (2009). Strain differences in taste aversion learning: Implications for animal models of drug abuse. In S. Reilly \& T. R. Schachtman (Eds.), Conditioned taste aversions: Behavioral and neural processes (pp. 226-261). New York: Oxford University Press.

Robertson, G. S., Pfaus, J. G., Atkinson, L. J., Matsumura, H., Phillips, A. G., \& Fibiger, H. C. (1991). Sexual behavior increases c-fos expression in the forebrain of the male rat. Brain Research, 564(2), 352-357.

Roma, P. G., Flint, W. W., Higley, J. D., \& Riley, A. L. (2006). Assessment of the aversive and rewarding effects of alcohol in Fischer and Lewis rats. Psychopharmacology (Berlin), 189(2), 187-199.

Shoaib, M., Schindler, C. W., \& Goldberg, S. R. (1997). Nicotine self-administration in rats: Strain and nicotine pre-exposure effects on acquisition. Psychopharmacology (Berlin), 129(1), $35-43$.

Siviy, S. M., Love, N. J., DeCicco, B. M., Giordano, S. B., \& Seifert, T. L. (2003). The relative playfulness of juvenile Lewis and Fischer-344 rats. Physiology \& Behavior, 80(2-3), 385-394.

Sternberg, E. M., Glowa, J. R., Smith, M. A., Calogero, A. E., Listwak, S. J., Aksentijevich, S., et al. (1992). Corticotropin releasing hormone related behavioral and neuroendocrine responses to stress in Lewis and Fischer rats. Brain Research, $570(1-2), 54-60$.

Sternberg, E. M., Hill, J. M., Chrousos, G. P., Kamilaris, T., Listwak, S. J., Gold, P. W., et al. (1989). Inflammatory mediator-induced hypothalamic-pituitary-adrenal axis activation is defective in streptococcal cell wall arthritis-susceptible Lewis rats. The Proceedings of the National Academy of Sciences Online (U. S.), 86(7), 2374-2378.

Straiko, M. M., Gudelsky, G. A., \& Coolen, L. M. (2007). Treatment with a serotonin-depleting regimen of MDMA prevents conditioned place preference to sex in male rats. Behavioral Neuroscience, 121(3), 586-593.

Suzuki, T., Otani, K., Koike, Y., \& Misawa, M. (1988). Genetic differences in preferences for morphine and codeine in Lewis and Fischer 344 inbred rat strains. Japanese Journal of Pharmacology, 47(4), 425-431.

Tang, X., Liu, X., Yang, L., \& Sanford, L. D. (2005). Rat strain differences in sleep after acute mild stressors and short-term sleep loss. Behavioural Brain Research, 160(1), 60-71.

Tzschentke, T. M. (1998). Measuring reward with the conditioned place preference paradigm: A comprehensive review of drug effects, recent progress and new issues. Progress in Neurobiology, 56(6), 613-672.

Tzschentke, T. M. (2007). Measuring reward with the conditioned place preference (CPP) paradigm: Update of the last decade. Addiction Biology, 12(3-4), 227-462.

Varty, G. B., \& Geyer, M. A. (1998). Effects of isolation rearing on startle reactivity, habituation, and prepulse inhibition in male Lewis, Sprague-Dawley, and Fischer F344 rats. Behavioral Neuroscience, 112(6), 1450-1457.

Westerman, A. T., Roma, P. G., Creed, E. T., Hurwitz, Z. E., \& Dominguez, J. M. (2009). The antiepileptic primidone impairs male rat sexual behavior. Pharmacology, Biochemistry and Behavior, 93(2), 160-164. 